SECTION 21. Pedagogy. Psychology. Innovations in the field of education.

\author{
Alexandr N. Shevtsov \\ candidate of Technical Sciences, \\ President, Theoretical \& Applied Science, LLP \\ associate Professor of the Department «Applied mathematics», \\ Taraz State University named after M.H. Dulati, Kazakhstan
}

Bazilbaeva Anastasiya Aleksandrovna

Primary school teacher, psychologist

Secondary school № 43, Taraz, Kazakhstan

\title{
METHOD OF THE USE AND DEVELOPMENT OF LABORATORY WORKS ON METHODS OF CALCULATIONS
}

Proposes a technique for carrying out of laboratory works on methods of calculations with the use of Delphi. Analysis of the proposed methodology.

Key words: Laboratory work, methods of calculations, methods of teaching.

\section{МЕТОДИКА ИСПОЛЬЗОВАНИЯ И РАЗРАБОТКА ЛАБОРАТОРНЫХ РАБОТ ПО МЕТОДАМ ВЫЧИСЛЕНИЙ.}

Предлагается методика проведения лабораторных работ по методам вычислений с применением Дельфи. Делается анализ предложенной методики.

Ключевые слова: Лабораторная работа, методы вычислений, методика преподавания.

В процессе преподавания дисциплины «Методы вычислений», для 3 курса бакалавриата специальности 5В060100 -«Математика», преподаватели как казахских так и русских отделений сталкиваются с проблемой выбора методики преподавания и алгоритмического языка для компьютерной реализации алгоритмов[1-2].

Построение методики должно включать как элементы теоретической подготовки, так и практического апробирования построенных моделей и формул, а также элементы самостоятельной работы и даже исследовательский аспект. Значит имеем следующую структурную схему:

1) теоретический материал,

2) постановка задачи из нескольких этапов,

3) компьютерная реализация алгоритмов 1 этапа, 
4) апробирование и отладка программы,

5) самостоятельное решение и реализация последующих этапов,

6) использование полученных навыков и алгоритмов в дальнейшей научно-исследовательской деятельности.

Рассмотрим примеры оформления лабораторных работ по темам «Решение СЛАУ по формулам Крамера», «Отделение корней. Метод половинного деления».

\begin{tabular}{|c|c|}
\hline $\begin{array}{l}\text { № стр. } \\
\text { схемы }\end{array}$ & Методический материал \\
\hline 1 & 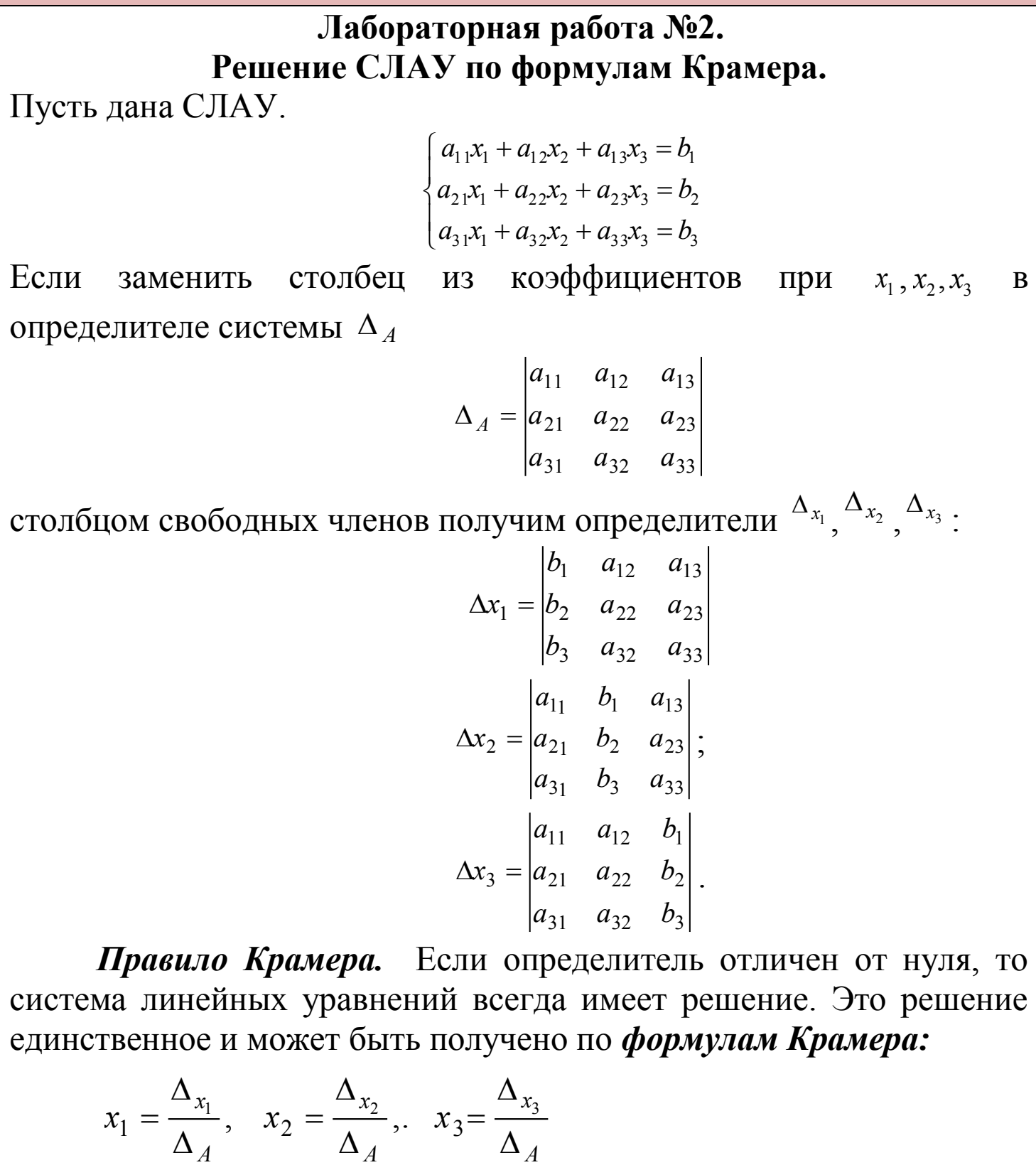 \\
\hline 2 & $\begin{array}{l}\text { Задание: Разработать компьютерную } \text { программу } \\
\text { решения СЛАУ люждения } \\
\text { любого порядка методом Крамера. }\end{array}$ \\
\hline 3 & $\begin{array}{l}\text { Решение } \\
\text { Запускаем Delphi, Создаем новое приложение VCL Form Aplication }\end{array}$ \\
\hline
\end{tabular}


Заполняем форму компонентами

StringGrid1

StringGrid2

Button1

Memo1

SpinEdit1

Label1 Label2 Label3 Label4

Создаем события

procedure Button1Click(Sender: TObject);

procedure SpinEdit1Change(Sender: TObject);

procedure FormCreate(Sender: TObject);

Окно программы:

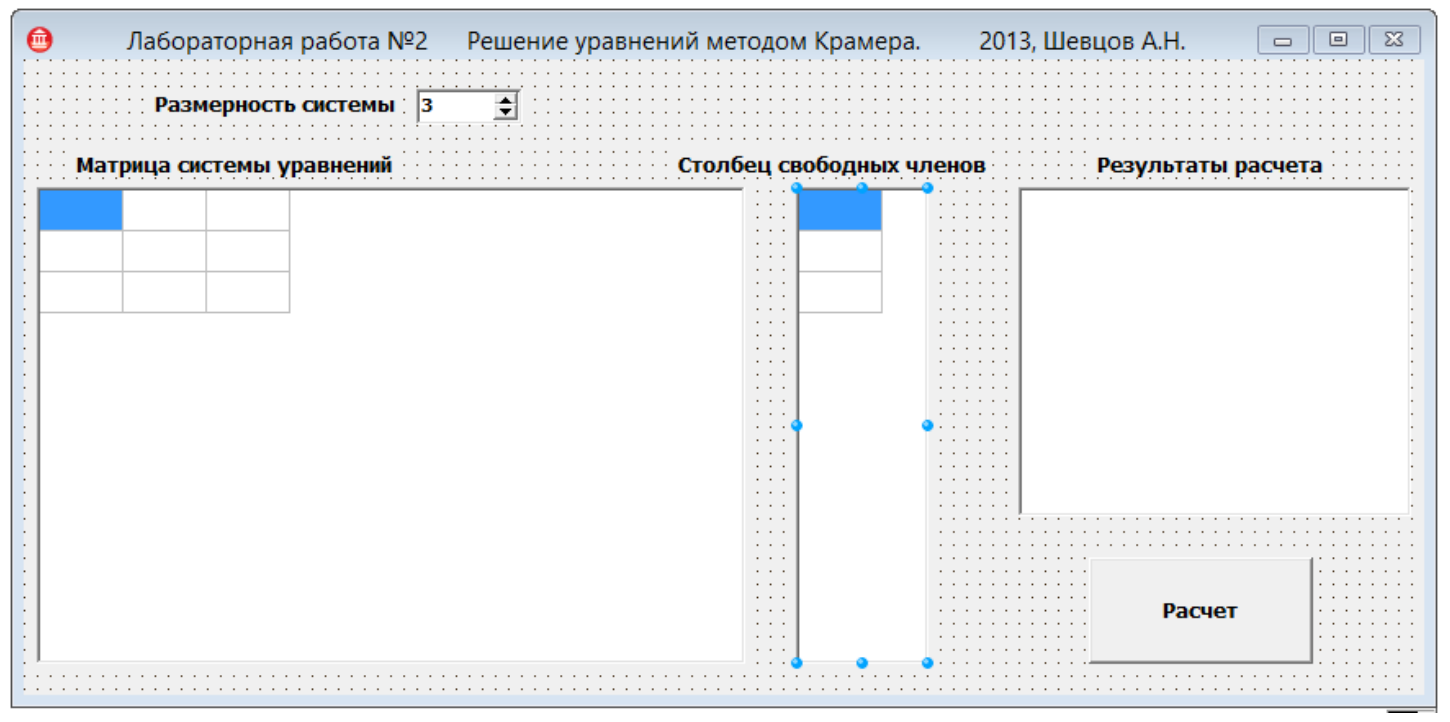

Создаем событие щелчок по кнопке «Расчет»

unit Unit1;

interface

uses

Windows, Messages, SysUtils, Variants, Classes, Graphics, Controls, Forms,

Dialogs, StdCtrls, Grids, Spin, Matrixes;

type

TForm1 $=$ class (TForm)

StringGrid1: TStringGrid;

StringGrid2: TStringGrid;

Button1: TButton;

Memo1: TMemo; 


\section{SpinEdit1: TSpinEdit;}

Label1: TLabel;

Label2: TLabel;

Label3: TLabel;

Label4: TLabel;

procedure Button1Click(Sender: TObject);

procedure SpinEdit1Change(Sender: TObject);

procedure FormCreate(Sender: TObject);

private

\{Private declarations \}

public

\{Public declarations

end;

type aa=array[1..10,1..10] of real;

var

Form1: TForm1;

\section{A:aa;}

B:array [1..10]of real;

$n, i, j$ :integer;

Delta:real;

implementation

$\left\{\$ R^{*} . \mathrm{dfm}\right\}$

function $\operatorname{det}(a: a a ; n:$ integer):real;

var $\mathrm{i}, \mathrm{j}$ :integer;

ax: TMatrix;

begin

ax := TMatrix.CreateE(n);

for $i:=1$ to $n$ do

for $\mathbf{j}:=1$ to $n$ do

ax.Items[i,j]:=a[i,j];

Det:=ax.Det;

ax.Free;

end;

procedure TForm1.Button1Click(Sender: TObject);

begin 


\begin{tabular}{|c|c|}
\hline & $\begin{array}{l}\text { n:=SpinEdit1.Value; } \\
\text { for } \mathrm{i}:=0 \text { to } n \text { - } 1 \text { do } \\
\text { for } \mathrm{j}:=0 \text { to } \mathrm{n}-1 \text { do } \\
\text { A[i+1,j+1]:=strtofloat(StringGrid1.Cells[i,j]); } \\
\text { for } \mathrm{i}:=0 \text { to } \mathrm{n} \text { - } 1 \text { do } \\
\text { B[i+1]:=strtofloat(StringGrid2.Cells[0,i]); } \\
\text { Delta:=det(A,n); } \\
\text { memo1.Lines.Add('det(A) = '+floattostr(Delta)); } \\
\text { end; } \\
\text { procedure TForm1.FormCreate(Sender: TObject); } \\
\text { begin } \\
\mathrm{n}:=10 ; \\
\text { for } \mathrm{i}:=0 \text { to } n \text { - } 1 \text { do } \\
\text { for } \mathrm{j}:=0 \text { to } n \text { - } 1 \text { do } \\
\text { StringGrid1.Cells[i,j]:=inttostr(random(5)); } \\
\text { for I := } 0 \text { to } n \text { - } 1 \text { do } \\
\text { StringGrid2.Cells[0,i]:=inttostr(random(5)); } \\
\text { end; } \\
\text { procedure TForm1.SpinEdit1Change(Sender: TObject); } \\
\text { begin } \\
\text { n:= SpinEdit1.Value; } \\
\text { stringgrid1.ColCount:=n; } \\
\text { stringgrid1.RowCount:=n; } \\
\text { stringgrid2.RowCount:=n; } \\
\text { end; } \\
\text { end. }\end{array}$ \\
\hline 4 & Получаем программу \\
\hline
\end{tabular}




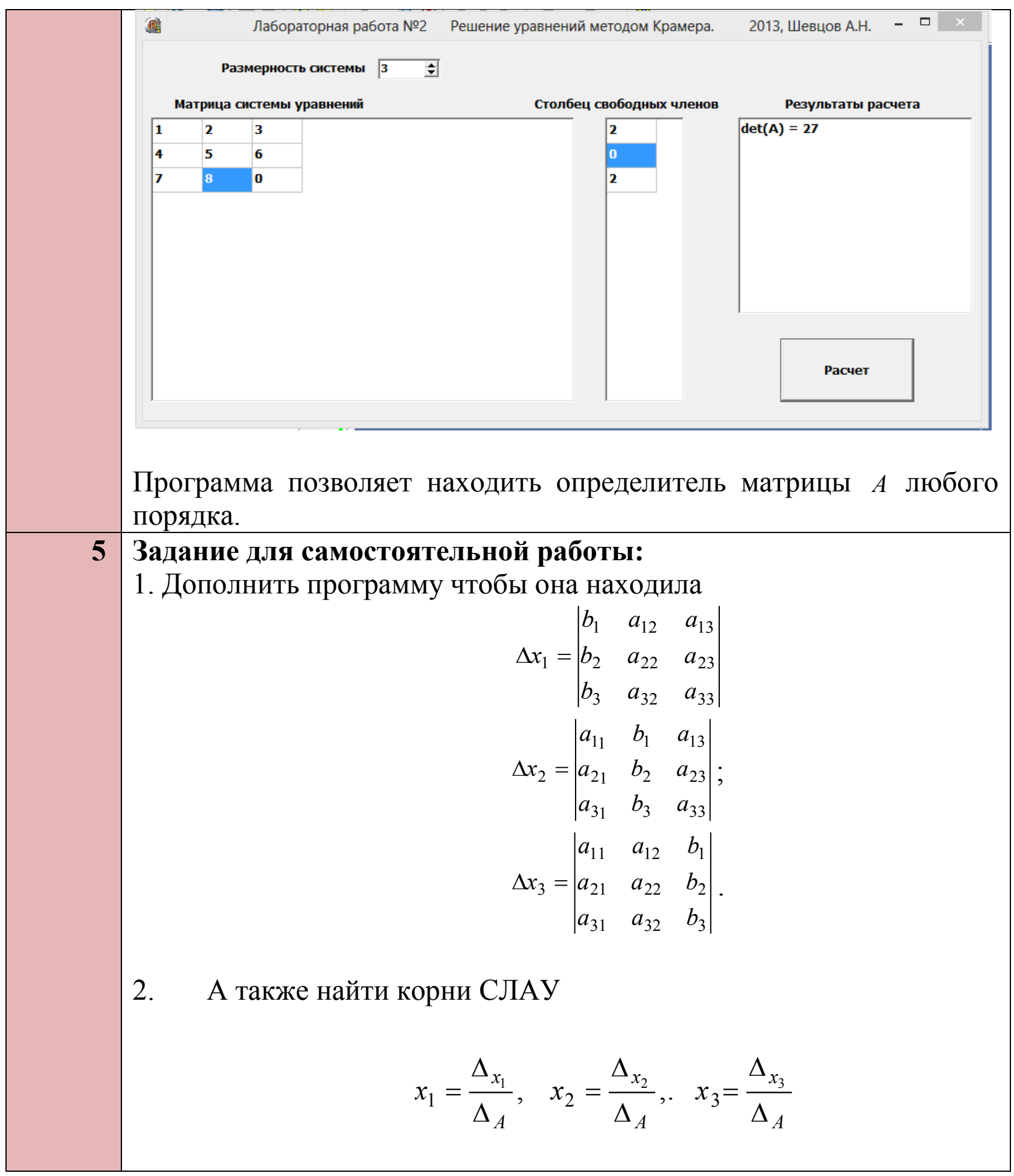

Рассмотрим еще один пример построения лабораторной работы

\begin{tabular}{|r|c|}
\hline $\begin{array}{r}\text { № стр. } \\
\text { схемы }\end{array}$ & Методический материал \\
\hline 1 & Лабораторная работа №3. \\
& Отделение корней \\
\hline
\end{tabular}




\begin{tabular}{|c|c|}
\hline & 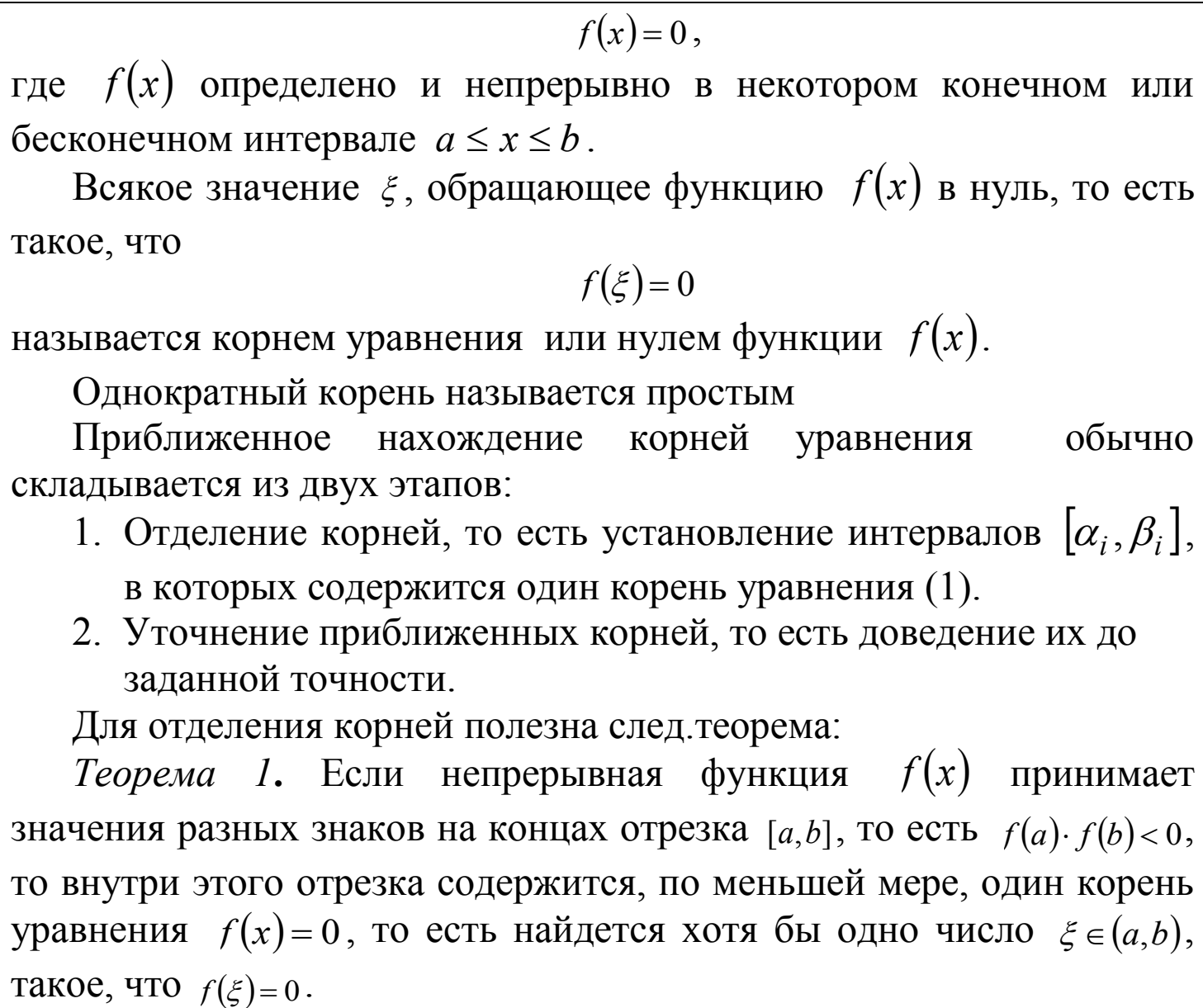 \\
\hline 2 & $\begin{array}{l}\text { Задание: Разработать компьютерную программу для отделения } \\
\text { корней. }\end{array}$ \\
\hline 3 & $\begin{array}{l}\text { Решение } \\
\text { Запускаем Delphi, Создаем новое приложение VCL Form Aplication } \\
\text { Заполняем форму компонентами } \\
\text { Edit1: TEdit; } \\
\text { Label1: TLabel; } \\
\text { Edit2: TEdit; } \\
\text { Label2: TLabel; } \\
\text { Edit3: TEdit; } \\
\text { Label3: TLabel; } \\
\text { Button1: TButton; } \\
\text { Memo1: TMemo; } \\
\text { Label4: TLabel; } \\
\text { Label5: TLabel; } \\
\text { Создаем coбытие } \\
\text { procedure Button1Click(Sender: TObject); } \\
\text { Окно программы: }\end{array}$ \\
\hline
\end{tabular}




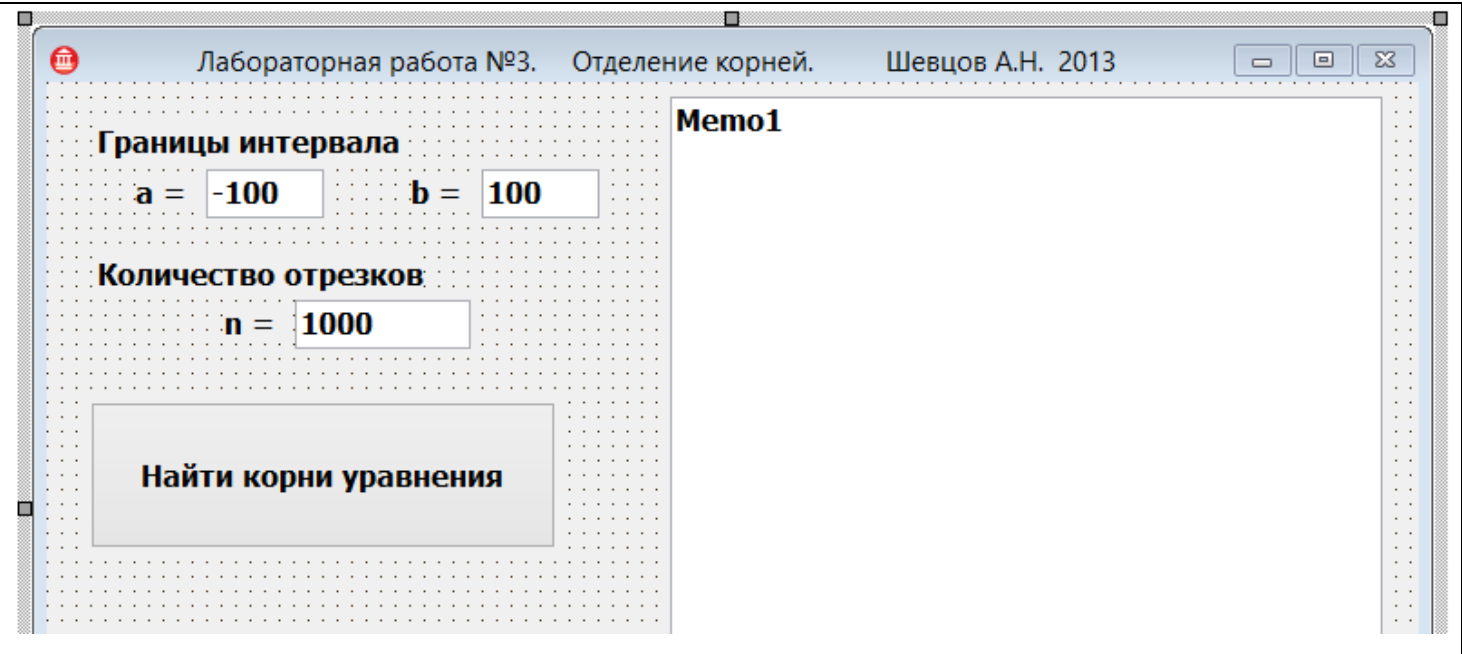

Создаем событие щелчок по кнопке «Расчет»

$$
\begin{aligned}
& \text { unit Unit1; } \\
& \text { interface } \\
& \text { uses }
\end{aligned}
$$

Windows, Messages, SysUtils, Variants, Classes, Graphics, Controls, Forms,

Dialogs, Math, StdCtrls;

type

TForm1 $=$ class $($ TForm)

Edit1: TEdit;

Label1: TLabel;

Edit2: TEdit;

Label2: TLabel;

Edit3: TEdit;

Label3: TLabel;

Button1: TButton;

Memo1: TMemo;

Label4: TLabel;

Label5: TLabel;

procedure Button1Click(Sender: TObject);

private

\{Private declarations \}

public

\{Public declarations \}

end;

var

Form1: TForm1;

$a, b, d x, x 1, x 2$ :real; 


\begin{tabular}{|c|c|}
\hline & $\begin{array}{l}\text { i,n:integer; } \\
\text { x:array[0..100000]of real; } \\
\text { implementation } \\
\left\{\$ R^{*} . d f m\right\} \\
\text { function } f(x: \text { real):real; } \\
\text { begin } \\
\text { f:=-power(x,7)+x*x+6; } \\
\text { end; } \\
\text { procedure TForm1.Button1Click(Sender: TObject); } \\
\text { begin } \\
\text { memo1.Clear; } \\
\text { a:=strtofloat(edit1.Text); } \\
\text { b:=strtofloat(edit2.Text); } \\
\text { n:=strtoint(edit3.Text); } \\
\text { dx:=(b-a)/n; } \\
\text { x[0]:=0; } \\
\text { for I := } 1 \text { to } n \text { do } \\
\text { begin } \\
\text { x[i]:=a+i*dx; } \\
\text { if f(x[i-1])*f(x[i])<0 then } \\
\text { begin } \\
x 1:=\text { round(x[i-1]*1000)/1000; } \\
\text { x2:=round(x[i]*1000)/1000; } \\
\text { memo1.Lines.Add('( '+floattostr(x1)+' ; '+floattostr(x2)+' )'); } \\
\text { end; } \\
\text { end; } \\
\text { if memo1.Text=" then memo1.Lines.Add('Kopнeй нeт'); } \\
\text { end; } \\
\text { end. }\end{array}$ \\
\hline 4 & $\begin{array}{l}\text { Получаем программу, которая позволяет отделять корни уравнения } \\
\text { определяя промежутки в которых они находятся. }\end{array}$ \\
\hline
\end{tabular}




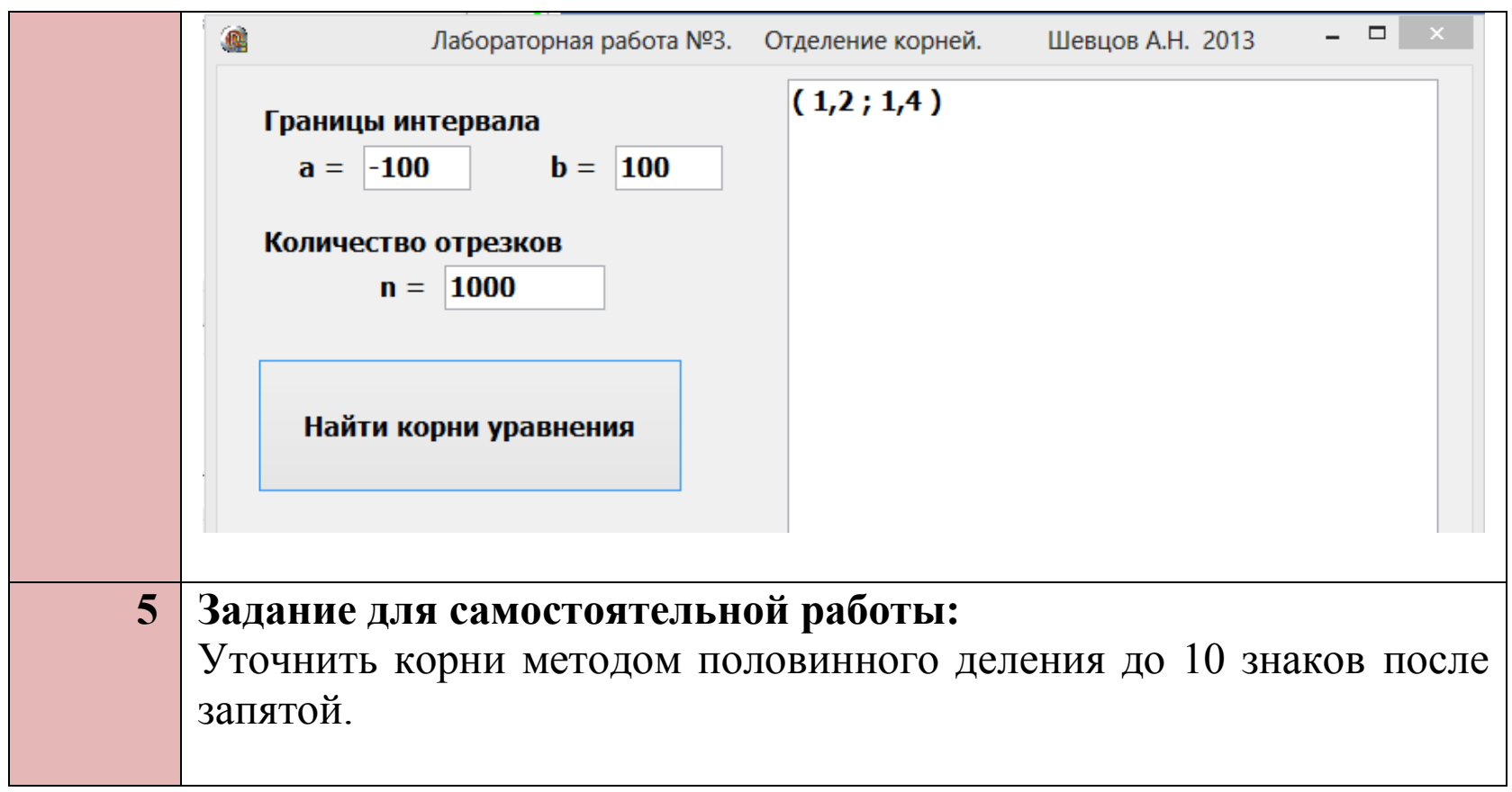

Здесь предполагается знание студентами основ программирования на языке Pascal, и умение строить небольшие алгоритмы. Хотя используемая методика позволяет на достаточно высоком уровне обучать и тех, кто ранее не сталкивался с программированием, и работой в Delphi.

На основе 3-х летнего опыта преподавания и проведения лабораторных работ непосредственно связанных с обучением студентов компьютерной реализацией алгоритмов, были получены следующие данные (табл.1).

\section{Таблица 1}

Процент студентов успешно справляющихся с заданием.

\begin{tabular}{|l|c|c|c|c|c|}
\hline \multirow{2}{*}{ № задания } & \multicolumn{5}{|c|}{ Лабораторные работы № } \\
\hline 1 & $\mathbf{1}$ & $\mathbf{2}$ & $\mathbf{3}$ & $\mathbf{4 - 1 0}$ & $\mathbf{1 0 - 1 5}$ \\
\hline 2 & - & - & - & - & - \\
\hline 3 & - & - & - & - & - \\
\hline 4 & $30 \%$ & $50 \%$ & $70 \%$ & $90 \%$ & $90 \%$ \\
\hline 5 & $5 \%$ & $20 \%$ & $30 \%$ & $70 \%$ & $80 \%$ \\
\hline
\end{tabular}

\section{Литература}

1. Медведев В.Г. Вычислительные методы: конспект лекций / Чуваш. ун-т. Чебоксары, 2006. 52c.

2. Nevzorov V. Delphi Russian Knowledge Base. -Chicago, USA. -2007, more than $4000 \mathrm{p}$. 